\title{
GEOMETRIES OF MATRICES. III. FUNDAMENTAL THEOREMS IN THE GEOMETRIES OF SYMMETRIC MATRICES
}

\author{
BY \\ LOO-KENG HUA
}

1. Introduction. The author gave, in the paper $I_{1}\left({ }^{1}\right)$, a discussion of the redundancy of the conditions which appeared in the generalization of von Staudt's theorem for symmetric matrices of order 2. The author then could also give a proof for matrices of all even orders but was unable to prove the result in its full generality, and further the proof depended essentially on some results about involutions. The author has now found a simpler proof which holds for any order greater than 2 and which is independent of the results about involutions. Nevertheless, the proof is carried out by means of mathematical induction upon the result of $I_{1}$, which depends essentially on the theory of involutions.

Several theorems for geometries keeping an involution as an absolute have also been obtained.

The second part of the paper is concerned with analytic mappings. The projective space of symmetric matrices may also be considered as the extended space of several complex variables as defined by Osgood( $\left.{ }^{2}\right)$. So far as the author is aware, the completeness of the group of automorphic mappings of an extended space has been established only for two special cases, namely the space of function theory and the complex projective space. If we assume that the group is topological, we may deduce the result from a theorem of E. Cartan $\left(^{3}\right)$ for semi-simple groups. In this paper, the problem is solved without any restriction besides analyticity.

As an application of the previous result in combination with the continuity theorem due to Levi $\left(^{4}\right)$ and with a result due to the author $\left.{ }^{5}\right)$, we solve also the corresponding problem for the group of automorphs of the elliptic space. It should be remarked that the corresponding problem for the hyperbolic space was solved by C. L. Siegel( $\left.{ }^{6}\right)$ in a recent important paper.

Presented to the Society, April 27, 1946; received by the editors May 30, 1945.

(1) Papers I, $I_{1}$, and II under the same title were published in Trans. Amer. Math. Soc. vol. 57 (1945) pp. 441-481, 482-490, vol. 61 (1947) pp. 193-228. They will be referred to in the present paper simply by their numbers.

(2) Lehrbuch der Funktionentheorie, vol. 2, 1929.

(3) La thérie des groupes finis et continus et l'analysis situs, Mémorial des sciences mathématiques, Paris, 1930, p. 51.

(4) E. E. Levi, Annales de Mathématiques Pures et Appliquées (3) vol. 17 (1910).

(5) L. K. Hua, Trans. Amer. Math. Soc. vol. 59 (1946) pp. 508-523.

(8) C. L. Siegel, Amer. J. Math. vol. 65 (1943) pp. 1-86. 
2. Arithmetic distance. Let $\subseteq$ and $\mathfrak{T}$ be two sets of points in the "projective" space of symmetric matrices. The arithmetic distance between $\mathfrak{S}$ and $\mathfrak{T}$ is defined to be the least upper bound of the arithmetic distance between any two points $S$ and $T$, where $S$ and $T$ belong to $\subseteq$ and $\mathfrak{I}$ respectively. It is denoted by $r(\mathfrak{S}, \mathfrak{T})$.

In particular for $\mathfrak{S}=\mathfrak{T}$, the distance $r(\mathfrak{S}, \mathfrak{S})$ is called the arithmetic diameter of the set $\mathfrak{S}$.

Given a positive integer $\rho$, a set $\subseteq$ is called a maximal set of rank $\rho$, if $\mathfrak{S}$ is of arithmetic diameter $\rho$, and if any set properly containing $\mathfrak{S}$ is of arithmetic diameter greater than $\rho$.

THEOREM 1. A normal subspace of rank $\rho$ is a maximal set of rank $\rho$.

Proof. Since arithmetic distance is invariant, we may take the normal subspace to consist of the points

$$
\left(\begin{array}{cc}
X^{(\rho)} & 0 \\
0 & 0
\end{array}\right)
$$

For any vector $v$ and any number $a$, not both zero, we have $X^{(\rho)}$ such that

$$
\left(\begin{array}{cc}
X^{(\rho)} & v^{\prime} \\
v & a
\end{array}\right)
$$

is a nonsingular $(\rho+1)$-rowed matrix. The theorem is now evident.

Is the converse of Theorem 1 true? It is true for $\rho=1$, but in general we have the following "gegenbeispiel":

The set of all matrices of rank 1 forms a maximal set $\subseteq$ of rank 2, but does not form a normal subspace. In fact, the equation

$$
d\left(\left(\begin{array}{lll}
x & y & z \\
y & u & v \\
z & v & w
\end{array}\right)-\left(\begin{array}{ccc}
a^{2} & a b & a c \\
a b & b^{2} & b c \\
a c & b c & c^{2}
\end{array}\right)\right)=0
$$

for all $a, b, c$ implies that

$$
\left(\begin{array}{lll}
x & y & z \\
y & u & v \\
z & v & w
\end{array}\right)
$$

is of rank 1. This fact shows that $\subseteq$ is a maximal set. On the other hand, we have $r(\Im, 0)=1$, which cannot hold in the normal subspace.

3. Arithmetic property of the normal subspace. Now we extend the concept of dieder manifold a little further:

Definition. Let $P_{1}$ and $P_{2}$ be two points. The points $X$ satisfying

$$
r\left(P_{1}, X\right)+r\left(X, P_{2}\right)=r\left(P_{1}, P_{2}\right)
$$


are said to form a dieder manifold spanned by $P_{1}$ and $P_{\mathbf{2}}$.

Theorem 2. A maximal set of rank $\rho$ is a normal space if and only if it contains all dieder manifolds spanned by any two points of the set.

Proof. (1) Let

$$
P=0, \quad Q=[1, \cdots, 1,0, \cdots, 0]
$$

(where the 1's are $\rho$ in number); we shall prove that, if

then $X$ takes the form

$$
r(P, X)+r(X, Q)=r(P, Q),
$$

$$
\left(\begin{array}{cc}
X_{1}^{(\rho)} & 0 \\
0 & 0
\end{array}\right) .
$$

We consider the pair of matrices $Q$ and $X$. There exists a nonsingular matrix $\Gamma\left({ }^{7}\right)$ such that

$$
\Gamma^{-1} X \Gamma^{\prime-1}=[1, \cdots, 1,0, \cdots, 0], \quad 0 \leqq p \leqq \rho
$$

(where the 1's are $p$ in number), and

$$
\Gamma^{-1} Q \Gamma^{\prime-1}=Q .
$$

From the second equation, we find that

Then

$$
\Gamma=\left(\begin{array}{ll}
A & B \\
0 & C
\end{array}\right), \quad A A^{\prime}=I .
$$

$$
X=\Gamma\left(\begin{array}{ll}
I^{(p)} & 0 \\
0 & 0
\end{array}\right) \Gamma^{\prime}
$$

Consequently, we have the assertion.

Therefore a normal subspace contains the dieder manifold spanned by any pair of points of the subspace.

(2) Without loss of generality, we may assume that the maximal set contains two points

$$
P=0, \quad Q=[1, \cdots, 1,0, \cdots, 0]
$$

(where the 1's are $\rho$ in number). So it contains

$$
P_{i}=[1, \cdots, 1,0,1, \cdots, 1,0, \cdots, 0], \quad 1 \leqq i \leqq \rho
$$

(where the first zero in the brackets appears in the $i$ th place, the second in the $(\rho-1)$ th), and

and so on.

$$
P_{i j}=P_{i} P_{j}, \quad P_{i j k}=P_{i} P_{j} P_{k},
$$

(7) See Turnbull and Aitken, Theory of canonical matrices, 1931, p. 135. 
Let

$$
X=\left(a_{r s}\right)_{1 \leqq r, s} n_{n}
$$

be a point of the set. Then

$$
r\left(X, P_{i}\right) \leqq \rho, \quad r\left(X, P_{i j}\right) \leqq \rho, \quad r\left(X, P_{i j k}\right) \leqq \rho,
$$

and so on. Consequently, the matrix

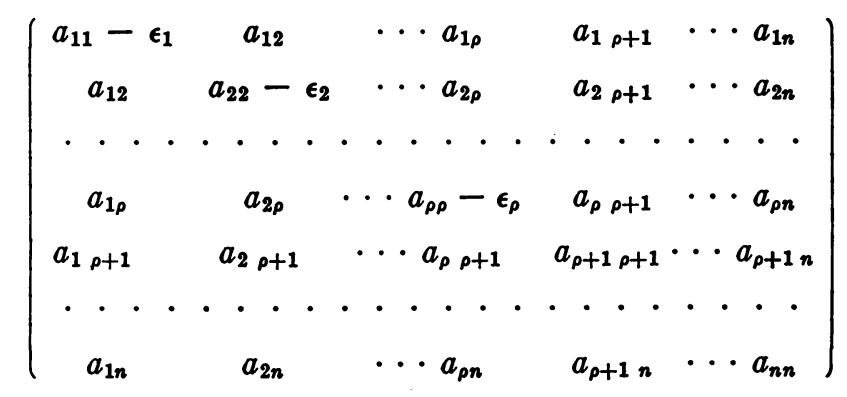

is always of rank $\rho$ for $\epsilon_{1}, \cdots, \epsilon_{\rho}$ arbitrarily taken from 0 and 1. In particular,

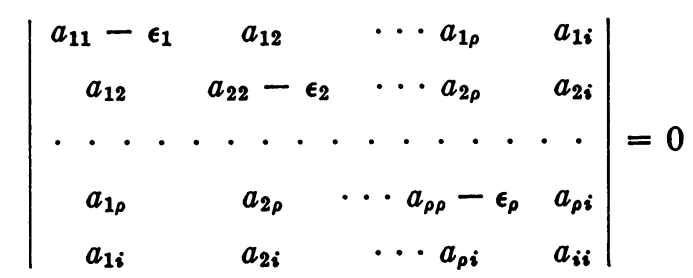

for $\rho<i \leqq n$ and any choice of $\epsilon$. Putting $\epsilon_{1}=0$ and $\epsilon_{1}=1$ and subtracting the results, we have

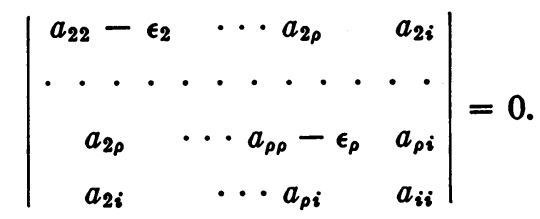

Repeating the same process, we have finally

$$
\left|\begin{array}{cc}
a_{\rho \rho}-\epsilon_{\rho} & a_{\rho i} \\
a_{\rho i} & a_{i i}
\end{array}\right|=0
$$

for $\epsilon_{\rho}=0$ and 1. Consequently $a_{i i}=0$ and $a_{\rho i}=0$. Varying $\rho$, we find that $a_{j i}=0$ for all $1 \leqq j \leqq \rho$. Further varying $i$ from $\rho+1$ to $n$, we have the assertion.

Therefore the elements of the set are of the form

$$
\left(\begin{array}{ll}
X^{(\rho)} & 0 \\
0 & 0
\end{array}\right)
$$

By Theorem 1 and (1) we have the second part of our theorem. 
Since pairs of points with arithmetic distance $\rho(1 \leqq \rho \leqq n)$ form a transitive set, the proof of the previous theorem establishes also the following:

THEOREM 3. Given two points with arithmetic distance $\rho$, there is one and only one normal subspace of rank $\rho$ which contains both.

\section{Proof of the fundamental theorem.}

Theorem 4. A continuous mapping carrying symmetric matrices into symmetric matrices and leaving arithmetic distance invariant is either a symplectic transformation or an anti-symplectic transformation.

Proof. (1) The theorem was proved for matrices of order 2 in $I_{1}$. Now we shall establish the general theorem by induction on the order of matrices. We thus suppose that the order of matrices is not less than 3 .

(2) Let

$$
\Gamma(Z)=Z_{1}
$$

be a mapping satisfying our conditions. The points of the form

$$
\left(\begin{array}{cc}
*{ }^{(n-1)} & 0 \\
0 & 0
\end{array}\right)
$$

form a normal subspace of rank $n-1$. Since the arithmetic distance is invariant, the set of points

$$
\Gamma\left(\begin{array}{cc}
* *^{(n-1)} & 0 \\
0 & 0
\end{array}\right)
$$

forms also a normal subspace of rank $n-1$. Since the totality of all normal subspaces of rank $n-1$ form a transitive set under the group of symplectic transformations, we may therefore assume that $\Gamma$ satisfies

$$
\Gamma\left(\begin{array}{cc}
\dot{W}^{(n-1)} & 0 \\
0 & 0
\end{array}\right)=\left(\begin{array}{cc}
W_{1}^{(n-1)} & 0 \\
0 & 0
\end{array}\right)
$$

It induces a continuous mapping on the $(n-1)$-rowed matrices $W$ and it keeps arithmetic distance invariant. Therefore by the hypothesis of induction we find either a symplectic mapping

$$
W=\left(\alpha W_{1}+\beta\right)\left(\gamma W_{1}+\delta\right)^{-1}, \quad \alpha=\alpha^{(n-1)},
$$

and so on, or an anti-symplectic mapping

$$
\bar{W}=\left(\alpha W_{1}+\beta\right)\left(\gamma W_{1}+\delta\right)^{-1} .
$$

The symplectic transformation $Z=\left(A Z_{1}+B\right)\left(C Z_{1}+D\right)^{-1}$ with

$$
A=\left(\begin{array}{ll}
\alpha & 0 \\
0 & 1
\end{array}\right), \quad B=\left(\begin{array}{ll}
\beta & 0 \\
0 & 0
\end{array}\right), \quad C=\left(\begin{array}{ll}
\gamma & 0 \\
0 & 0
\end{array}\right), \quad D=\left(\begin{array}{ll}
\delta & 0 \\
0 & 1
\end{array}\right)
$$


carries the transformation $\Gamma$ inducing (3) into a new one satisfying

$$
\Gamma\left(\begin{array}{cc}
W^{(n-1)} & 0 \\
0 & 0
\end{array}\right)=\left(\begin{array}{cc}
W^{(n-1)} & 0 \\
0 & 0
\end{array}\right)
$$

Applying the same method to those $\Gamma$ inducing (4), we conclude, in both cases, that we may assume, without loss of generality, that $\Gamma$ satisfies (5).

(3) Since

$$
\Gamma\left(\begin{array}{cc}
0^{(n-1)} & 0 \\
0 & 1
\end{array}\right)
$$

is a matrix of rank 1 , we may let

$$
\Gamma\left(\begin{array}{cc}
0^{(n-1)} & 0 \\
0 & 1
\end{array}\right)=\left(a_{1}, \cdots, a_{n}\right)^{\prime}\left(a_{1}, \cdots, a_{n}\right) .
$$

Since the arithmetic distance between

$$
\left(\begin{array}{cc}
I^{(n-1)} & 0 \\
0 & 0
\end{array}\right), \quad\left(\begin{array}{cc}
0^{(n-1)} & 0 \\
0 & 1
\end{array}\right)
$$

is equal to $n$, we have $a_{n} \neq 0$. The transformation $Z_{1}=A^{\prime} Z A$ with

$$
A=\left(\begin{array}{cc}
I^{(n-1)} & 0 \\
-v a_{n}^{-1} & a_{n}^{-1}
\end{array}\right), \quad v=\left(a_{1}, \cdots, a_{n-1}\right),
$$

carries (5) into itself and $\left(a_{1}, \cdots, a_{n}\right)^{\prime}\left(a_{1}, \cdots, a_{n}\right)$ into

$$
\left(\begin{array}{cc}
0^{(n-1)} & 0 \\
0 & 1
\end{array}\right)
$$

Therefore, we may assume further that

$$
\Gamma\left(\begin{array}{cc}
0^{(n-1)} & 0 \\
0 & 1
\end{array}\right)=\left(\begin{array}{cc}
0^{(n-1)} & 0 \\
0 & 1
\end{array}\right) .
$$

(4) The transformation $\Gamma$ leaves

$$
\left(\begin{array}{lll}
0^{(1)} & 0 & 0 \\
0 & I^{(n-2)} & 0 \\
0 & 0 & 0
\end{array}\right), \quad\left(\begin{array}{lll}
0 & 0 & 0 \\
0 & 0^{(n-2)} & 0 \\
0 & 0 & 1
\end{array}\right)
$$

invariant, therefore, by Theorem 2 , we find

$$
\Gamma\left(\begin{array}{ll}
0 & 0 \\
0 & W^{(n-1)}
\end{array}\right)=\left(\begin{array}{ll}
0 & 0 \\
0 & W_{1}^{(n-1)}
\end{array}\right) .
$$

By the supposition of induction, we have either 


$$
W^{(n-1)}=\left(\alpha W_{1}+\beta\right)\left(\gamma W_{1}+\delta\right)^{-1}
$$

or

$$
\bar{W}^{(n-1)}=\left(\alpha W_{1}+\beta\right)\left(\gamma W_{1}+\delta\right)^{-1},
$$

where

$$
\left(\begin{array}{ll}
\alpha & \beta \\
\gamma & \delta
\end{array}\right)
$$

is a $2(n-1)$-rowed symplectic matrix.

According to (5), the transformation (7) and (8) leaves every point of the form

$$
W=\left(\begin{array}{ll}
X^{(n-2)} & 0 \\
0 & 0
\end{array}\right)
$$

invariant; (8) cannot hold.

Since (7) leaves every point of the form (9) invariant, we have

$$
\begin{array}{ll}
\alpha=\left(\begin{array}{cc} 
\pm I^{(n-2)} & a_{2}^{\prime} \\
0 & a_{4}
\end{array}\right), & \beta=0 \\
\gamma=\left(\begin{array}{ll}
0 & c_{2}^{\prime} \\
c_{3} & c_{4}
\end{array}\right), & \delta=\left(\begin{array}{ll} 
\pm I^{(n-2)} & 0 \\
\mp a_{2} a_{4}^{-1} & a_{4}^{-1}
\end{array}\right) .
\end{array}
$$

Further, (7) carries

$$
\left(\begin{array}{ll}
0 & 0 \\
0 & 1^{(1)}
\end{array}\right)
$$

into itself; we have $a_{2}=0$.

Let

$$
\begin{array}{ll}
A=\left(\begin{array}{cc} 
\pm 1 & 0 \\
0 & \alpha^{(n-1)}
\end{array}\right), & B=\left(\begin{array}{ll}
0 & 0 \\
0 & \beta^{(n-1)}
\end{array}\right), \\
C=\left(\begin{array}{ll}
0 & 0 \\
0 & \gamma^{(n-1)}
\end{array}\right), & D=\left(\begin{array}{cc} 
\pm 1 & 0 \\
0 & \delta^{(n-1)}
\end{array}\right) .
\end{array}
$$

The transformation

$$
Z=\left(A Z_{1}+B\right)\left(C Z_{1}+D\right)^{-1}
$$

carries $\Gamma$ into a new one satisfying (5) and

$$
\Gamma\left(\begin{array}{ll}
0 & 0 \\
0 & W^{(n-1)}
\end{array}\right)=\left(\begin{array}{ll}
0 & 0 \\
0 & W^{(n-1)}
\end{array}\right)
$$

since the transformation leaves every point

$$
\left(\begin{array}{ll}
X_{1}^{(n-1)} & 0 \\
0 & 0
\end{array}\right)
$$


invariant.

(5) Since

$$
\Gamma\left(\begin{array}{ll}
1 & 0 \\
0 & 0^{(n-1)}
\end{array}\right)=\left(\begin{array}{ll}
1 & 0 \\
0 & 0^{(n-1)}
\end{array}\right), \quad \Gamma\left(\begin{array}{ll}
0^{(n-1)} & 0 \\
0 & 1
\end{array}\right)=\left(\begin{array}{ll}
0^{(n-1)} & 0 \\
0 & 1
\end{array}\right),
$$

we have

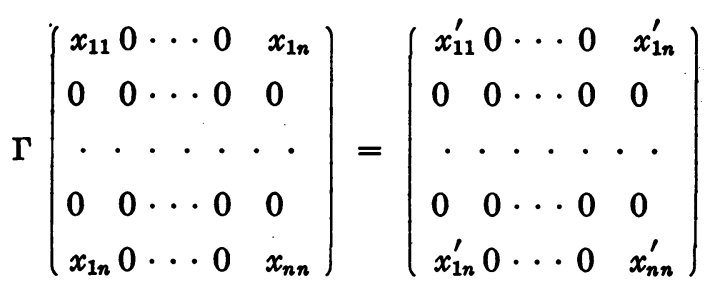

and by hypothesis of induction, we have

$$
\left(\begin{array}{ll}
x_{11} & x_{1 n} \\
x_{1 n} & x_{n n}
\end{array}\right)=\left(\alpha\left(\begin{array}{cc}
x_{11}^{\prime} & x_{1 n}^{\prime} \\
x_{1 n}^{\prime} & x_{n n}^{\prime}
\end{array}\right)+\beta\right)\left(\gamma\left(\begin{array}{cc}
x_{11}^{\prime} & x_{1 n}^{\prime} \\
x_{1 n}^{\prime} & x_{n n}^{\prime}
\end{array}\right)+\delta\right)^{-1},
$$

which is a symplectic transformation. (Notice that we omit the "anti-symplectic" case by the same reason given in (3).)

Since it leaves every point

$$
\left(\begin{array}{ll}
x & 0 \\
0 & 0
\end{array}\right), \quad\left(\begin{array}{ll}
0 & 0 \\
0 & y
\end{array}\right)
$$

invariant, we have either

$$
\alpha=I^{(2)}, \quad \beta=0, \quad \gamma=\left(\begin{array}{ll}
0 & c \\
c & 0
\end{array}\right), \quad \delta=I^{(2)}
$$

or

$$
\alpha=\left(\begin{array}{rr}
1 & 0 \\
0 & -1
\end{array}\right), \quad \beta=0, \quad \gamma=\left(\begin{array}{rr}
0 & c \\
-c & 0
\end{array}\right), \quad \delta=\left(\begin{array}{rr}
1 & 0 \\
0 & -1
\end{array}\right)
$$

For the first case the transformation with

$$
A=I, \quad B=0, \quad C=\left(\begin{array}{ccccc}
0 & 0 & \cdots & 0 & c \\
0 & 0 & \cdots & 0 & 0 \\
\cdots & \cdots & . & . & . \\
0 & 0 & \cdots & 0 & 0 \\
c & 0 & \cdots & 0 & 0
\end{array}\right), \quad D=I
$$


and for the second case the transformation with

$$
A=[1,1, \ldots, 1,-1], \quad B=0, \quad C=\left(\begin{array}{ccccc}
0 & 0 & \cdots & 0 & c \\
0 & 0 & \cdots & 0 & 0 \\
\cdots & \cdots & \cdots & . \\
0 & 0 & \cdots & 0 & 0 \\
-c & 0 & \cdots & 0 & 0
\end{array}\right), \quad D=A,
$$

carry $\Gamma$ into a new one which satisfies (5), (10) and

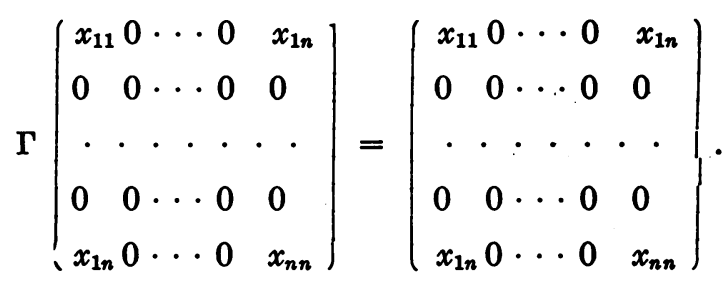

(6) In particular, we conclude that we may assume that

and

$$
\begin{gathered}
\Gamma\left(\left[\lambda_{1}, \cdots, \lambda_{n-1}, 0\right]\right)=\left[\lambda_{1}, \cdots, \lambda_{n-1}, 0\right], \\
\Gamma\left(\left[0, \lambda_{2}, \cdots, \lambda_{n}\right]\right)=\left[0, \lambda_{2}, \cdots, \lambda_{n}\right],
\end{gathered}
$$

$$
\Gamma\left(\left[\lambda_{1}, 0, \cdots, 0, \lambda_{n}\right]\right)=\left[\lambda_{1}, 0, \cdots, 0, \lambda_{n}\right] .
$$

Now we are going to prove that

$$
\Gamma\left(\left[\lambda_{1}, \cdots, \lambda_{n}\right]\right)=\left[\lambda_{1}, \cdots, \lambda_{n}\right] .
$$

Since

$$
r\left(\left[\lambda_{1}, \cdots, \lambda_{n}\right],\left[\lambda_{1}, \cdots, \lambda_{n-1}, 0\right]\right)=1
$$

we have

$$
\Gamma\left(\left[\lambda_{1}, \cdots, \lambda_{n}\right]\right)=\left[\lambda_{1}, \cdots, \lambda_{n-1}, 0\right]+\left(a_{1}, \cdots, a_{n}\right)^{\prime}\left(a_{1}, \cdots, a_{n}\right) .
$$

Since

$$
r\left(\left[\lambda_{1}, \cdots, \lambda_{n}\right],\left[0, \lambda_{2}, \cdots, \lambda_{n}\right]\right)=1 \text {, }
$$

we have $a_{2}=a_{3}=\cdots=a_{n-1}=0$. Further, since

$r\left(\left[\lambda_{1}, \lambda_{2}, \cdots, \lambda_{n}\right],\left[\lambda_{1}, 0, \cdots, 0, \lambda_{n}\right]\right)$

$=r\left(\left[\lambda_{1}, \cdots, \lambda_{n-1}, 0\right]+\left(a_{1}, 0, \cdots, 0, a_{n}\right)^{\prime}\left(a_{1}, 0, \cdots, 0, a_{n}\right),\left[\lambda_{1}, 0, \cdots, 0, \lambda_{n}\right]\right)$,

we obtain that the matrix

$$
\left(\begin{array}{cc}
a_{1}^{2} & a_{1} a_{n} \\
a_{1} a_{n} & a_{n}^{2}-\lambda_{n}
\end{array}\right)
$$

is of rank zero, that is $a_{1}=0$ and $a_{n}^{2}=\lambda_{n}$. Thus we have the assertion. 
(7) Let

$$
\Gamma(Z)=Z_{1}, \quad Z=\left(z_{i j}\right), \quad Z_{1}=\left(z_{i j}^{\prime}\right) .
$$

Since (12) holds, the equation

$$
d\left(Z-\left[\lambda_{1}, \cdots, \lambda_{n}\right]\right)=0
$$

(consider $Z$ as fixed and vary $\lambda_{1}, \cdots, \lambda_{n}$ ) implies

$$
d\left(Z_{1}-\left[\lambda_{1}, \cdots, \lambda_{n}\right]\right)=0
$$

and vice versa. This implies

$$
d\left(Z-\left[\lambda_{1}, \cdots, \lambda_{n}\right]\right)=d\left(Z_{1}-\left[\lambda_{1}, \cdots, \lambda_{n}\right]\right)
$$

identically in $\lambda_{1}, \cdots, \lambda_{n}$. Consequently, we have

$$
z_{i i}=z_{i i}^{\prime}, \quad z_{i i} z_{j j}-z_{i j}^{2}=z_{i i}^{\prime} z_{j j}^{\prime}-z_{i j}^{\prime 2},
$$

and

$$
z_{i j}= \pm z_{i j}^{\prime}
$$

The transformation

$$
[1,-1,1, \cdots, 1] Z[1,-1,1, \cdots, 1]
$$

carries $z_{i i}$ into itself and $z_{12}$ into $-z_{12}$. Thus we may assume that

$$
z_{1 i}=z_{1 i}^{\prime} \text {. }
$$

By the same consideration given in the proof of Theorem 8 of $I$, we have

$$
\Gamma(Z)=Z \text {. }
$$

The theorem therefore follows.

5. Affine geometry of symmetric matrices.

THEOREM 5. A continuous mapping carrying finite points into finite points, infinite points into infinite points, and keeping arithmetic distance invariant is either an affine mapping

$$
W=A Z A^{\prime} r S
$$

or an anti-affine mapping

$$
\bar{W}=A Z A^{\prime}+S .
$$

This is an immediate consequence of Theorem 4.

6. Möbius geometry of symmetric matrices. As in II, we let $\Im$ be a fundamental involution as an absolute, for example:

$$
W=H Z H,
$$


where $H=[1,1, \cdots, 1,-1]$. The arithmetic distance between $P$ and $\Im(P)$ is either 0 or 2. In case $P=\Im(P)$ we say that $P$ is a point-matrix.

THEOREM 6. A continuous mapping carrying point-matrices into point-matrices, non-point-matrices into non-point-matrices and keeping arithmetic distance invariant is a Möbius mapping or an anti-Möbius mapping.

Proof. We take $\Im$ in the form of (14), which carries matrices of the form

$$
\left(\begin{array}{cc}
Z_{1}^{(n-1)} & 0 \\
0 & z
\end{array}\right)
$$

into themselves. Thus the mappings under consideration carry the set defined by (15) onto itself.

Without loss of generality we may assume that the mappings keep 0 and $\infty$ invariant; by Theorem 4 , it takes either the form

$$
W=A Z A^{\prime}
$$

or

$$
\bar{W}=A Z A^{\prime} \text {. }
$$

For the first case, we put

and we have

$$
A=\left(\begin{array}{ll}
A_{1} & \alpha_{1}^{\prime} \\
\alpha_{2} & a
\end{array}\right)
$$

$$
\left(\begin{array}{cc}
W_{1} & 0 \\
0 & w
\end{array}\right)=\left(\begin{array}{ll}
A_{1} & \alpha_{1}^{\prime} \\
\alpha_{2} & a
\end{array}\right)\left(\begin{array}{ll}
Z_{1} & 0 \\
0 & z
\end{array}\right)\left(\begin{array}{cc}
A_{1}^{\prime} & \alpha_{2}^{\prime} \\
\alpha_{1} & a
\end{array}\right) .
$$

Consequently, we have

$$
A_{1} Z_{1} \alpha_{2}^{\prime}+\alpha_{1}^{\prime} z a=0
$$

for all $Z_{1}$ and $z$. It follows that

$$
\alpha_{1}=0 \text { or } \quad a=0
$$

and

$$
\alpha_{2}=0 \text { or } A_{1}=0 \text {. }
$$

The case with $\alpha_{1}=0, \alpha_{2}=0$ is what we are looking for. The other cases cannot happen by the nonsingularity of $A$, except when $n=2, a=A_{1}=0$. For this case we have also a Möbius mapping.

A similar method may be used for the second case.

7. Manifold at infinity. Let $M$ be an idempotent symmetric matrix, that is, $M^{2}=M$. Then $I-M$ is also an idempotent symmetric matrix, since $(I-M)^{2}=I-2 M+M^{2}=I-M$. Further $M$ and $I-M$ annihilate each other, namely

$$
M(I-M)=(I-M) M=0 .
$$


From an idempotent symmetric matrix $M$, we can construct a symplectic transformation

$$
W=(M Z+(I-M))(-(I-M) Z+M)^{-1} .
$$

Now we consider those transformations with diagonal $M$. They are $2^{n}$ in number, including the identity. The $2^{n}-1$ non-identity transformations are called the fundamental semi-involutions of the space of symmetric matrices. They are of fundamental importance in the study of the extended space.

For the sake of later use, we give the explicit expression of the semiinvolution with

Let

$$
M=\left(\begin{array}{ll}
I^{(r)} & 0 \\
0 & 0
\end{array}\right)
$$

we have

$$
Z=\left(\begin{array}{cc}
Z_{11}^{(r)} & Z_{12} \\
Z_{12}^{\prime} & Z_{22}
\end{array}\right)
$$

$$
W=\left(\begin{array}{cc}
Z_{11}-Z_{12} Z_{22}^{-1} Z_{12}^{\prime} & -Z_{12} Z_{22}^{-1} \\
-Z_{22}^{-1} Z_{12}^{\prime} & -Z_{22}^{-1}
\end{array}\right) .
$$

THEOREM 7. Every point at infinity can be carried into a finite point by one of the $2^{n}-1$ semi-involutions.

Proof. Suppose that

$$
\left(Z_{1}, Z_{2}\right)
$$

be the homogeneous coordinate of a point at infinity, with $Z_{2}$ of rank $r$. There exist two permutation matrices( $\left.{ }^{8}\right) P$ and $Q$ such that

$$
P Z_{2} Q=\left(\begin{array}{ll}
Z_{11}^{(r)} & * \\
* & *
\end{array}\right)
$$

where $Z_{11}^{(r)}$ is nonsingular. We have a nonsingular $R$ such that

Let

$$
R P Z_{2} Q=\left(\begin{array}{cc}
Z_{11} & Z_{12} \\
0 & 0
\end{array}\right)
$$

Then

$$
R P\left(Z_{1}, Z_{2}\right)\left(\begin{array}{ll}
Q^{\prime-1} & 0 \\
0 & Q
\end{array}\right)=\left(W_{1}, W_{2}\right)
$$

( 8 ) Corresponding to a permutation

$$
\left(\begin{array}{l}
1,2, \cdots, n \\
i_{1}, i_{2}, \cdots, i_{n}
\end{array}\right)
$$

we have a permutation matrix which is the matrix of the linear transformation $x_{p}^{\prime}=x_{i p}$ $(1 \leqq p \leqq n)$. Evidently, we have $P^{\prime}=P^{-1}$. 
Let

$$
W_{2}=\left(\begin{array}{cc}
Z_{11} & Z_{12} \\
0 & 0
\end{array}\right)
$$

and

$$
W_{1}=\left(\begin{array}{ll}
W_{11} & W_{12} \\
W_{21} & W_{22}
\end{array}\right)
$$

We have immediately

$$
T=\left(\begin{array}{cc}
I & -Z_{11}^{-1} Z_{12} \\
0 & I
\end{array}\right) .
$$

and

$$
W_{2} T=\left(\begin{array}{ll}
Z_{11} & 0 \\
0 & 0
\end{array}\right)
$$

$$
W_{1} T^{\prime-1}=\left(\begin{array}{cc}
* & * \\
0 & W_{22}
\end{array}\right) .
$$

The zero at the left lower corner is obtained because $\left(W_{1}, W_{2}\right)$ is a symmetric pair. Then $W_{22}$ is nonsingular, since $\left(W_{1}, W_{2}\right)$ is a nonsingular pair.

Let

Then

$$
M=\left(\begin{array}{ll}
I^{(r)} & 0 \\
0 & 0
\end{array}\right)
$$

where

$$
\left(W_{1}, W_{2}\right)\left(\begin{array}{cc}
M & I-M \\
-(I-M) & M
\end{array}\right)=\left(P_{1}, P_{2}\right),
$$

$$
P_{1}=W_{1}(I-M)+W_{2} M=\left(\begin{array}{cc}
0 & W_{12} \\
0 & W_{22}
\end{array}\right)+\left(\begin{array}{cc}
Z_{11} & Z_{12} \\
0 & 0
\end{array}\right),
$$

which is evidently nonsingular.

Now we consider

$$
\begin{array}{r}
\left(\begin{array}{ll}
Q & 0 \\
0 & Q
\end{array}\right)\left(\begin{array}{cc}
M & I-M \\
-(I-M) & M
\end{array}\right)\left(\begin{array}{ll}
Q^{\prime} & 0 \\
0 & Q^{\prime}
\end{array}\right)=\left(\begin{array}{cc}
M_{1} & I-M_{1} \\
-\left(I-M_{1}\right) & M_{1}
\end{array}\right), \\
M_{1}=Q M Q^{-1},
\end{array}
$$

which is a semi-involution, since $Q^{\prime}=Q^{-1}$. The theorem is therefore established.

Since

$$
d(-(I-M) Z+M)
$$

is a principal minor of $Z$, we have the following theorem.

THEOREM 8. The manifold at infinity is carried by the $2^{n}-1$ semi-involutions into manifolds defined by equating the principal minors of $Z$ to zero. 
8. Group of analytic automorphs of the space. Now we come to the second part of the paper. The projective space of symmetric matrices may be regarded as an extended space of several complex variables as defined by Osgood. The aim of the present section is to establish the following theorem.

THEOREM 9. An analytic mapping carrying the extended space onto itself is a symplectic mapping.

Proof. (1) Let

$$
W=\Gamma(Z)
$$

be an analytic mapping carrying the projective space of symmetric matrices onto itself. By a theorem due to Osgood $\left({ }^{(}\right)$, the mapping is birational; consequently, (18) may be written as

$$
w_{i j}=p_{i j}(Z) / q(Z)
$$

where $p_{i j}(Z)$ and $q(Z)$ are $2^{-1} n(n+1)+1$ polynomials without common divisor.

(2) There is a point $S$ at which

$$
p_{i j}(S) \neq 0, \quad q(S) \neq 0 .
$$

The transformation $Z=Z_{1}+S$ carries (18) into a new one with

$$
p_{i j}(0) \neq 0, \quad q(0) \neq 0 .
$$

The transformation

$$
W=\Gamma\left(-Z_{1}^{-1}\right)
$$

maps also the space onto itself, and

$$
w_{i j}=p_{i j}\left(-Z_{1}^{-1}\right)\left(d\left(Z_{1}\right)\right)^{\lambda} / q\left(-Z_{1}^{-1}\right)\left(d\left(Z_{1}\right)\right)^{\lambda},
$$

where $\lambda$ is the least integer making all numerators and denominators integral.. By (20), $p_{i j}\left(-Z_{1}^{-1}\right)\left(d\left(Z_{1}\right)\right)^{\lambda}$ and $q\left(-Z_{1}^{-1}\right)\left(d\left(Z_{1}\right)\right)^{\lambda}$ are of degree $n \lambda$. Consider the Jacobian of (21). Let $\Delta$ and $\Delta_{1}$ be the inverses of the Jacobians of (18) and (21) respectively. Notice that $\Delta$ and $\Delta_{1}$ are polynomials, for otherwise there would exist some point for which the Jacobians vanish. Then we have

$$
\Delta_{1}\left(Z_{1}\right)=\Delta\left(-Z_{1}^{-1}\right)\left(d\left(Z_{1}\right)\right)^{n+1},
$$

since the Jacobian of $Z=-Z_{1}^{-1}$ is $\left(d\left(Z_{1}\right)\right)^{-(n+1)}$. Since $q(0) \neq 0$, we have $\Delta(0)$ $\neq 0$. Consequently $\Delta_{1}\left(Z_{1}\right)$ is a polynomial of degree $n(n+1)$.

Without loss of generality, we may now assume that $p_{i j}$ and $q$ are polynomials of degree $n \lambda$ and that the inverse of the Jacobian of (18) is a polynomial of degree $n(n+1)$ and its term of highest degree is equal to a constant

(?) Ibid. p. 295. 
multiple of $(d(Z))^{n+1}$. The highest terms of $p_{i j}$ and $q$ are also constant multiples of $(d(Z))^{\lambda}$.

(3) We decompose $q$ into irreducible factors

$$
q=q_{1}^{\lambda_{1}} \cdots q_{l}^{\lambda_{l}}
$$

Now we are going to establish that $\left(q_{1} \cdots q_{\imath}\right)^{n+1}$ divide $\Delta$. By some easy transformation we may suppose that $q_{1}$ does not divide $p_{n n}$. We consider the product of the transformation (16) with $r=n-1$ and (18). The inverse of the Jacobian of the new mapping is equal to

$$
\left(p_{n n} / q\right)^{n+1} \Delta,
$$

which is a polynomial. Thus $q_{1}{ }^{n+1}$ divides $\Delta$. Therefore we have the assertion.

(4) Since $d^{\prime} Z$ ) is irreducible and

$$
\Delta(Z)=c(d(Z))^{n+1}+\cdots,
$$

we have immediately that $l=1$. Therefore

$$
q(Z)=(a d(Z)+\cdots)^{\lambda} .
$$

Further (16) with $(n-1)$ carries $p_{n n}$ into the denominator, then we have also

$$
p_{n n}(Z)=\left(a_{n n} d(Z)+\cdots\right)^{\lambda} .
$$

The mapping cannot be one-to-one except when $\lambda=1$.

Now we may assume that

$$
\begin{aligned}
q(Z) & =a d(Z)+\cdots, \\
p_{i j}(Z) & =a_{i j} d(Z)+\cdots,
\end{aligned}
$$

and

$$
\Delta(Z)=c(q(Z))^{n+1} .
$$

(5) Now we shall prove that $p_{i i} p_{i j}-p_{i j}{ }^{2}$ is divisible by $q$. In fact, by (16) with $r=n-2$, we have

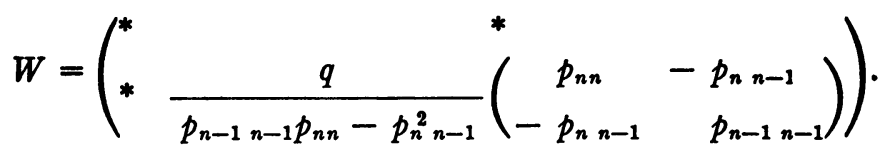

If $q$ does not divide $p_{n-1} n-1 p_{n n}-p_{n}^{2}{ }_{n-1}$, the manifold $q=0$ is mapped into a manifold of dimension not greater than $n(n+1)-3$, which is impossible. Similarly, every three-rowed principal minor of $\left(p_{i j}\right)$ is divisible by $q$.

(6) Since

$$
\Delta\left(-Z^{-1}\right)(d(Z))^{n+1}=\Delta_{1}(Z),
$$

we have

$$
q\left(-Z^{-1}\right)(d(Z))=q_{1}(Z)
$$


where $q_{1}(Z)$ is a polynomial. By means of $(16)$ with $r=n-1$, we may find that

$$
p_{i i}\left(-Z^{-1}\right) d(Z)
$$

is also a polynomial. Further, by means of (16) with $r=n-2$, we find that

$$
\frac{p_{i i}\left(-Z^{-1}\right) p_{i j}\left(-Z^{-1}\right)-p_{i j}^{2}\left(-Z^{-1}\right)}{q\left(-Z^{-1}\right)} d(z)
$$

is also a polynomial. Thus

$$
p_{i j}^{2}\left(-Z^{-1}\right)(d(Z))^{2}
$$

is a polynomial, and so is $p_{i j}\left(-Z^{-1}\right) d(Z)$. Therefore we have to find the polynomial $p(Z)$ of degree $n$ such that

$$
p\left(-Z^{-1}\right) d(Z)
$$

is a polynomial.

(7) The answer to the question raised in (6) is that if we put

$$
p(Z)=\sum_{k=0}^{n} p^{(k)}(Z)
$$

where $p^{(k)}$ is a homogeneous polynomial of degree $k$, then $p^{(k)}(Z)$ is a linear combination of the $k$-rowed minors of $Z$. This will be proved in the next section, owing to its independent interest.

(8) Now we have, instead of (23) and (24), the following expressions

$$
\begin{aligned}
q(Z) & =a d(Z)+\sum_{k=0}^{n-1} q^{(k)}, & a \neq 0, \\
p_{i j}(Z) & =a_{i j} d(Z)+\sum_{k=0}^{n-1} p_{i j}^{(k)}, &
\end{aligned}
$$

where $q^{(k)}$ and $p_{i j}^{(k)}$ are linear combinations of the $k$-rowed minors of $Z$. We may let $a=1$. Let $z_{i j}{ }^{*}$ be the cofactor of $z_{i j}$, then

$$
q^{(n-1)}=\sum_{1 \leqq i \leqq j \leqq n} c_{i j} z_{i j}^{*}
$$

There exists $S$ such that

$$
q(Z-S)
$$

contains no term of order $n-1$. Thus, we may assume that $q^{(n-1)}=0$.

Further the transformation

$$
X=W-\left(a_{i j}\right)
$$

carries (18) into a new one with $a_{i j}=0$. Up to the present, (18) takes the form 


$$
q(Z)=d(Z)+\sum_{k=0}^{n-2} q^{(k)}
$$

and

$$
p_{i j}(Z)=\sum_{k=0}^{n-1} p_{i j}^{(k)},
$$

where $q^{(k)}$ and $p_{i j}{ }^{(k)}$ are linear combinations of the $k$-rowed minors of $Z . \Delta$ is given by (25).

Since the singular matrices form a manifold of dimension $n(n+1)-2$, there is a nonsingular matrix $Z$ carried into a nonsingular matrix $W$. Without loss of generality we may assume that

$$
I=\Gamma(I) .
$$

(9) We write

$$
p_{i j}^{(n-1)}=\sum_{1 \leqq s \leqq t \leqq n} a_{i j, s t} z_{s t}^{*} . \quad 1 \leqq i ; j \leqq n .
$$

We shall prove that (29) forms a system of independent equations. In fact, the degree of the Jacobian of (18) with (26) and (27) is, by direct verification, not greater than

$$
(-2) \frac{n(n+1)}{2}-1=-n(n+1)-1 .
$$

the last " -1 " appears, as the highest terms are dependent. Then $\Delta$ is of degree not less than $n(n+1)+1$, which is impossible. We have therefore the assertion.

(10). Now we consider the matrix

$$
\left(p_{i j}^{(n-1)}\right)=\sum_{1 \leqq s, t \leqq n} A_{s t} z_{s t}^{*}
$$$$
A_{s t}^{\prime}=A_{s t}
$$

By (5), we have the consequence that $d(Z)$ divides

$$
\left|\begin{array}{ll}
p_{i i}^{(n-1)} & p_{i j}^{(n-1)} \\
p_{i j}^{(n-1)} & p_{j i}^{(n-1)}
\end{array}\right| \text { and }\left|\begin{array}{lll}
p_{i i}^{(n-1)} & p_{i j}^{(n-1)} & p_{i k}^{(n-1)} \\
p_{i j}^{(n-1)} & p_{i j}^{(n-1)} & p_{j k}^{(n-1)} \\
p_{i k}^{(n-1)} & p_{j k}^{(n-1)} & p_{k k}^{(n-1)}
\end{array}\right| .
$$

Therefore if $d(Z)=0$, we find that (30) is of rank not greater than 1. In particular $A_{i i}$ are of rank 1 , as that $A_{i i}$ cannot be of rank 0 has been shown in (9).

We write

$$
A_{i i}=\left(a_{i 1}, \cdots, a_{i n}\right)^{\prime}\left(a_{i 1}, \cdots, a_{i n}\right) .
$$

By (28), we have 
Let

$$
\sum_{i=1}^{n} A_{i i}=I
$$

then

$$
A=\left(a_{i j}\right)
$$

$$
A^{\prime} A=I \text {. }
$$

Thus $A \Gamma(Z) A^{\prime}$ carries (30) into a new one with

$$
A_{i i}=[0, \cdots, 0,1,0, \cdots, 0]
$$

(where there are $i-1$ zeros preceding the one).

We write

$$
p_{11}^{(n-1)}=z_{11}^{*}+\sum_{1 \leq i<j \leq n} a_{i j} z_{i j}^{*}, \quad p_{22}^{(n-1)}=z_{22}^{*}+\sum_{1 \leqq i<j \leq n} c_{i j} z_{i j}^{*}
$$

Since $d(Z)$ divides

$$
p_{11}^{(n-1)} p_{22}^{(n-1)}-\left(p_{12}^{(n-1)}\right)^{2}
$$

we write

$$
p_{11}^{(n-1)} p_{22}^{(n-1)}-\left(p_{12}^{(n-1)}\right)^{2}=d(Z) g(Z) .
$$

By the lemma given in the next section, $p_{11}^{(n-1)} p_{22}^{(n-1)}-\left(p_{12}^{(n-1)}\right)^{2}$ is a linear combination of the two-rowed minors of

$$
\left(z_{i j}^{*}\right) \text {. }
$$

We write

$$
p_{12}^{(n-1)}=\sum_{1 \leqq i<j \leqq n} b_{i j} z_{i j}^{*}
$$

If $p_{12}$ contains $z_{i j}{ }^{*}(i j \neq 1,2)$ then $p_{11} p_{22}$ must contain $x_{i i}{ }^{*} x_{j j}{ }^{*}$, which is impossible. Therefore

$$
p_{12}^{(n-1)}= \pm z_{12}^{*}
$$

Consequently,

$$
p_{11}^{(n-1)} p_{22}^{(n-1)}-\left(p_{12}^{(n-1)}\right)^{2}=z_{11}^{*} \sum c_{i j}^{*} z_{i j}^{*}+z_{i j}^{*} \sum a_{i j} z_{i j}^{*}+\left(\sum a_{i j} z_{i j}^{*}\right)\left(\sum c_{i j} z_{i j}^{*}\right)
$$

is a multiple of $d(Z)$. This is possible only when $c=a=0$. Thus we have

$$
p_{i i}^{(n-1)}=z_{i i}^{*}, \quad p_{i j}^{(n-1)}= \pm z_{i j}^{*} .
$$

The transformation

$$
[1,-1,1, \cdots, 1] Z^{*}[1,-1, \cdots, 1]
$$

carries $z_{12}{ }^{*}$ into $-z_{12}{ }^{*}$, therefore we may assume that 


$$
p_{1 i}^{(n-1)}=z_{1 i}^{*}
$$

Since

$$
\left|\begin{array}{ccc}
z_{11}^{*} & z_{12}^{*} & z_{13}^{*} \\
z_{12}^{*} & z_{22}^{*} & -z_{23}^{*} \\
z_{18}^{*} & -z_{23}^{*} & z_{33}^{*}
\end{array}\right|
$$

is not divisible by $d(Z)$, we have

$$
p_{i j}^{(n-1)}=z_{i j}^{*}
$$

Up to the present, we arrive at the conclusion that we may let

$$
q(Z)=d(Z)+\sum_{k=0}^{n-2} q^{(k)}
$$

and

$$
p_{i j}(z)=z_{i j}^{*}+\sum_{k=0}^{\dot{n}-2} p_{i j}^{(k)}
$$

(11) We write

$$
p_{11} p_{22}-p_{12}^{2} \equiv 0(\bmod q)
$$

in a more precise form

$$
p_{11} p_{22}-p_{12}^{2}=q \psi
$$

where

$$
\psi=\psi^{(n-2)}+\psi^{(n-3)}+\cdots,
$$

and $\psi^{(k)}$ is a homogeneous polynomial of degree $k$.

Comparing the terms of degree $2 n-3$ in (33), we have

$$
z_{11}^{*} p_{22}^{(n-2)}+z_{22}^{*} p_{11}^{(n-2)}-2 z_{12}^{*} p_{12}^{(n-2)}=d(Z) \psi^{(n-8)} \text {. }
$$

By the result which will be proved in (12) we have

$$
p_{11}^{(n-2)}=p_{12}^{(n-2)}=p_{22}^{(n-2)}=\psi^{(n-3)}=0 .
$$

Now we are going to prove that $p_{11}{ }^{(\sigma)}, p_{12}{ }^{(\sigma)}, p_{22}{ }^{(\sigma)}$ and $\psi^{(\sigma-1)}$ are all zero. Suppose that the assertion is true for $\sigma>\rho$. Then

$$
z_{11}^{*} p_{22}^{(\rho)}+z_{22}^{*} p_{11}^{(\rho)}-2 z_{12}^{*} p_{12}^{(\rho)}=d(Z) \psi^{(\rho-1)} \text {. }
$$

By (12) we have $p_{11}^{(\rho)}=p_{12}^{(\rho)}=p_{22}^{(\rho)}=\psi^{(\sigma-1)}=0$. Thus we have finally

$$
q(Z)=d(Z), \quad p_{i j}(Z)=z_{i j}^{*} .
$$


The theorem is proved completely, except the verification of the following assertion.

(12) For $n-2 \geqq \sigma>0$, from

$$
z_{11}^{*} p_{22}^{(\sigma)}+z_{22}^{*} p_{11}^{(\sigma)}-2 z_{12}^{*} p_{12}^{(\sigma)}=d(Z) \psi^{(\sigma-1)},
$$

we can deduce that $p_{11}^{(\sigma)}=p_{12}^{(\sigma)}=p_{22}^{(\sigma)}=\psi^{(\sigma-1)}=0$.

To prove this assertion, we make a transformation $Z=W^{-1}$. Then

$$
\begin{gathered}
z_{i j}^{*}=w_{i j} / d(W), \quad p_{i j}^{(\sigma)}(Z)=r_{i j}^{(n-\sigma)}(W) / d(W), \\
\psi^{(\sigma-1)}(Z)=\phi^{(n-\sigma+1)}(W) / d(W) ;
\end{gathered}
$$

we have

$$
w_{11} r_{22}^{(n-\sigma)}+w_{22} r_{11}^{(n-\sigma)}-2 w_{12} r_{12}^{(n-\sigma)}=\phi^{(\tau)}(W),
$$

where $\phi^{(\tau)}$ is a sum of $\tau=(n-\sigma+1)$-rowed minors. Let $\phi_{1}$ be a $\tau$-rowed minor contained in $\phi$. Putting all elements of $W$ other than those contained in $\phi$ equal to zero, we find that $\phi_{1}$, a determinant of order $\tau$, may be expressed".as

$$
w_{12} r_{22}+w_{22} r_{11}-2 w_{12} r_{12}
$$

where $r_{11}, r_{12}, r_{22}$ are sums of $(\tau-1)$-rowed minors of $\phi_{1}$. This is impossible for $n>3$ as shown by the fact that the determinant vanishes identically for $w_{11}=w_{12}=w_{22}=0$. Thus we suppose that $\tau=3$. Since $w_{22}$ contains no term with factors $w_{11}$ and $w_{13}$, we have

$$
r_{22}=c_{1}\left(w_{22} w_{33}-w_{23}^{2}\right),
$$

and similarly

$$
r_{11}=c_{2}\left(w_{11} w_{33}-w_{13}^{2}\right) .
$$

The equality

$$
\begin{aligned}
c_{1}\left(w_{22} w_{33}-w_{23}^{2}\right) w_{11}+c_{2}\left(w_{11} w_{33}-w_{13}^{2}\right) & w_{22}-2 w_{12} r_{12} \\
& =w_{11} w_{22} w_{33}-w_{23}^{2} w_{11}-w_{13}^{2} w_{22}+\cdots
\end{aligned}
$$

implies

$$
\begin{aligned}
c_{1}\left(w_{22} w_{33}-w_{23}^{2}\right) w_{11}+c_{2}\left(w_{11} w_{33}-w_{13}\right)^{2} w_{23} & \\
& \equiv w_{11} w_{22} w_{33}-w_{23}^{2} w_{11}-w_{13}^{2} w_{22}\left(\bmod w_{12}\right),
\end{aligned}
$$

which is evidently impossible.

9. A result concerning adjugate. Now we are going to verify the assertion stated in (7) of the previous section.

Let

$$
X=\left(x_{i j}\right)
$$


denote an $n$-rowed symmetric matrix and let

$$
Y=\left(y_{i j}\right)
$$

be its inverse. Now we consider the following problem: find the polynomial $f(Y)$ in $y_{i j}$ satisfying

$$
f(Y) d(X)=g(X),
$$

where $g(X)$ is a polynomial in $x_{i j}$. Since $d(X)$ is a homogeneous polynomial, we need only consider the homogeneous part of $f(Y)$ as well as $g(X)$. Thus our problem is reduced to finding homogeneous $f(Y)$ satisfying (34). Let $l$ be the degree of $f(Y)$ and

$$
f(Y)=\sum_{p_{11}+\cdots+p_{n n}=l} p\left(p_{11}, \cdots, p_{n n}\right) y_{11}^{p_{11}} \cdots y_{n n}^{p_{n n}} .
$$

Then $g(X)$ is of degree $n-l$ and can be written as

$$
g(X)=\sum_{q_{11}+\cdots+q_{n n}=n-l} q\left(q_{11}, \cdots, q_{n n}\right) x_{11}^{q_{11}} \cdots x_{n n}^{q_{n n}} .
$$

Let

$$
X^{*}=\left(x_{i j}^{*}\right)=d(X) Y .
$$

Then (34) may be written as

$$
\begin{aligned}
\sum_{p_{11}+\cdots+p_{n n}=k} p\left(p_{11}, \cdots, p_{n n}\right) x_{11}^{* p_{11}} \cdots x_{n n}^{* p_{n n}} & \\
& =(d(X))^{l-1} \sum_{q_{11}+\cdots+q_{n n}-n-l} q\left(q_{11}, \cdots, q_{n n}\right) x_{11}^{q_{11}} \cdots x_{n n}^{q_{n n}} .
\end{aligned}
$$

Notice that the relation (37) is a reciprocal one, in fact, from (37), we deduce also

$$
\begin{aligned}
\sum_{q_{11}+\cdots+q_{n n}=n-l} q\left(q_{11}, \cdots, q_{n n}\right) x_{11}^{* q_{11}} \cdots x_{n n}^{* q_{n n}} & \\
& =d(X)^{n-l-1} \sum_{p_{11}+\cdots+p_{n n}=n} p\left(p_{11}, \cdots, p_{n n}\right) x_{11}^{p_{11}} \cdots x_{n n}^{p_{n n}} .
\end{aligned}
$$

Does (37) have a solution? It is known $\left({ }^{10}\right)$ that a minor of order $l$ satisfies our requirement. The purpose of the present section is to establish the converse, namely:

THEOREM 10. (37) holds if and only if (36) is a linear combination of the $(n-l)$-rowed minors. Consequently, (34) holds if and only if $g(X)$ is a linear combination of the minors of $X$.

${ }^{(10)}$ Wedderburn, Lectures on matrices, Amer. Math. Soc. Colloquium Publications, vol. 17, p. 67 , formula (20). 
Proof. The theorem is evidently true for $l=0,1, n$ and $n-1$. Consequently the theorem is true for $n=1,2$ and 3 .

Let $n \geqq 4$ and $n / 2 \leqq l \leqq n-2$. We write

$$
g(X)=g_{1}(X)+g_{0}(X),
$$

where $g_{1}(X)$ vanishes for $x_{11}=x_{12}=\cdots=x_{1 n}=0$. Putting

$$
X=\left(\begin{array}{ll}
1 & 0 \\
0 & X_{1}
\end{array}\right) \text {, }
$$

we find, by comparing the homogeneous parts, that $g_{0}(X)=g_{0}\left(X_{1}\right)$ satisfies (37) with $l-1, n-1$ instead of $l, n$. By hypothesis of induction, $g_{0}(X)$ is a linear combination of $(n-l)$-rowed minors of $X_{1}$.

Let

$$
g_{1}(X)=g_{2}(X)+g_{1,0}(X)
$$

where $g_{2}(X)$ vanishes for

$$
x_{11}=x_{12}=\cdots=x_{1 n}=0
$$

and for

$$
x_{12}=x_{22}=\cdots=x_{2 n}=0 .
$$

Then $g_{10}(X)$ is also a linear combination of the $(n-l)$-rowed minors of $X$.

Proceeding successively, we have

$$
g(X)=g_{0}(X)+g_{10}(X)+\cdots+g_{n 0}(X)+\psi(X),
$$

where $\psi(X)$ vanishes for

for

$$
x_{11}=x_{12}=\cdots=x_{1 n}=0 \text {, }
$$$$
x_{12}=x_{22}=\cdots=x_{2 n}=0 \text {, }
$$$$
\text { ............... }
$$

and for

$$
x_{1 n}=x_{2 n}=\cdots=x_{n n}=0 .
$$

Since the degree of $\psi(X)$ is $n-l \leqq n / 2$, it is only possible for $l=n / 2$, and $\psi(X)$ contains only terms of the form

$$
c x_{12} x_{34} \cdots x_{n-1 n} .
$$

The term $\left(x_{11} \cdots x_{n n}\right)^{l-1}\left(x_{12} x_{34} \cdots x_{n-1 n}\right)$ cannot appear on the left side of (37). Therefore we have the theorem.

10. Elliptic geometry. Now we let

$$
\xi=\left(\begin{array}{ll}
H & 0 \\
0 & H
\end{array}\right)
$$


where $H=[1, \cdots, 1,-1, \cdots,-1]$ with $p$ positive 1 's and $q$ negative 1 's, $p+q=n$.

The points $\left(W_{1}, W_{2}\right)$ making

$$
\left(\overline{W_{1}, W_{2}}\right) \xi\left(W_{1}, W_{2}\right)^{\prime}
$$

of the same signature as $H$ form the elliptic space with signature $(p, q)$. The symplectic transformation

$$
\left(Z_{1}, Z_{2}\right)=Q\left(W_{1}, W_{2}\right) F, \quad \bar{F} \xi F^{\prime}=\xi
$$

is called a motion of the space.

THEOREM 11. Any nonsingular symmetric pair of matrices $\left(W_{1}, W_{2}\right)$ making (43) nonsingular belongs to the elliptic space of signature $(p, q)$.

Proof. We have a nonsingular matrix $\Gamma$ such that

$$
\left.\bar{\Gamma} \overline{\left(W_{1}, W_{2}\right.}\right) \xi\left(W_{1}, W_{2}\right)^{\prime} \Gamma^{\prime}=H_{0}
$$

where $H_{0}$ is a diagonal matrix with $p^{\prime}$ positive 1's and $q^{\prime}$ negative 1 's.

Let

$$
\left(W_{1}^{*}, W_{2}^{*}\right)=\Gamma\left(W_{1}, W_{2}\right) .
$$

Construct the symplectic matrix

$$
F=\left(\begin{array}{cc}
W_{1}^{*} & W_{2}^{*} \\
-H_{0} \bar{W}_{2}^{*} H & H_{0} \bar{W}_{1}^{*} H
\end{array}\right) .
$$

We may verify directly that

$$
\bar{F}\left(\begin{array}{ll}
H & 0 \\
0 & H
\end{array}\right) F^{\prime}=\left(\begin{array}{ll}
H_{0} & 0 \\
0 & H_{0}
\end{array}\right) .
$$

Owing to the invariance of signature, we have $H=H_{0}$. The theorem follows.

From Theorem 11, we have that the elliptic space of signature $(p, q)$ is formed by all nonsingular symmetric pairs of matrices $\left(W_{1}, W_{2}\right)$ except those lying on

$$
d\left(\left(\overline{W_{1}, W_{2}}\right) \xi\left(W_{1}, W_{2}\right)^{\prime}\right)=0 .
$$

From Theorem 11, we may easily find that

$$
d\left(\left(\overline{W_{1}, W_{2}}\right) \xi\left(W_{1}, W_{2}\right)^{\prime} H\right) \geqq 0
$$

for any nonsingular pair of matrices $\left(W_{1}, W_{2}\right)$ and that the equality holds on a manifold of dimension not greater than $n(n+1)-2$. Now we shall go further to establish the following theorem. 
THEOREM 12. If

$$
d\left(\left(\overline{W_{1}, W_{2}}\right) \xi\left(W_{1}, W_{2}\right)^{\prime}\right)=0
$$

then $\left(W_{1}, W_{2}\right)$ lies on a manifold of dimension not greater than $n(n+1)-3$.

Proof. By the consideration of semi-involutions, we have only to consider the nonhomogeneous expression, that is, we are going to prove that the symmetric matrices $Z$ satisfying

$$
d(H+Z H \bar{Z})=0
$$

form a manifold of dimension not greater than $n(n+1)-3$.

The equation (46) implies that $H Z^{-1} H \bar{Z}^{-1}$ has at least a negative root, if $Z$ is nonsingular. By a theorem due to the author( $\left.{ }^{11}\right)$, those $Z$ form a manifold of dimension not greater than $n(n+1)-3$.

The equation $d(Z)=0$ does not imply $d(H+Z H \bar{Z})=0$ identically. The manifold defined by

$$
d(Z)=d(H+Z H \bar{Z})=0
$$

is therefore of dimension not greater than $n(n+1)-3$.

Theorem 13 (Fundamental theorem of the elliptic space). An analytic automorph of the elliptic space of signature $(p, q)$ is a motion of the space.

Proof. Let

$$
W=\Gamma(Z), \quad \Gamma=\left(f_{i j}\right)
$$

by an analytic mapping of the elliptic space onto itself. Then $f_{i j}(Z)$ is analytic in the whole extended space of symmetric matrices except possibly on a manifold of dimension not greater than $n(n+1)-3$. By the continuity theorem( $\left.{ }^{12}\right)$ of functions of several complex variables, $f_{i j}(Z)$ is analytic in the whole extended space. Its invcrse mapping is also analytic everywhere. The theorem follows from Theorem 12.

11. Hyperbolic space. The hyperbolic space is formed by the symmetric matrices $Z$ making $I-Z \bar{Z}$ positive definite. A symplectic mapping carrying the space onto itself is called a motion of the space.

Theorem 14 (SIEGel). An analytic mapping carrying the hyperbolic space onto itself is a motion of the space.

For completeness, we give here a proof which is different from that due to Siegel.

Proof. (1) The space is transitive, hence we need only to consider the trans-

(11) Ibid. Theorem 6.

(12) Levi, ibid., or Satz 17, Folgerung 1 of Behnke and Thullen, Theorie der Funktionen mehrerer komplexer Veränderlichen, Julius Springer, 1934. 
formations keeping 0 invariant. By a theorem for circular regions proved by $\mathrm{H}$. Cartan $\left({ }^{13}\right)$, the mapping is a linear one. Let

$$
W=\sum_{1 \leqq r \leqq s \leqq h} A_{r s} z_{r s}, \quad A_{r s}=A_{r s}^{\prime},
$$

be the mapping. Since $I-Z \bar{Z}=0$ is the intersection of all the algebraic surfaces bounding the space, the mapping (47), therefore, carries unitary symmetric matrices into unitary symmetric matrices.

Since any unitary symmetric matrix can be expressed as $U U^{\prime}$ where $U$ is unitary, we may assume that (47) carries $I$ into itself. Consequently, we have

$$
\sum_{r=1}^{n} A_{r r}=I
$$

Putting $Z=\left[e^{i \theta_{1}}, \cdots, e^{i \theta_{n}}\right]$, we have

$$
I=W \bar{W}=\left(\sum_{r=1}^{n} A_{r r} e^{i \theta_{r}}\right)\left(\sum_{r=1}^{n} \bar{A}_{r r} e^{-i \theta_{r}}\right)
$$

for any real $\theta$. Consequently we obtain

$$
A_{r r} \bar{A}_{s s}=0 \quad \text { for } r \neq s .
$$

(3) From (48) and (49), we have

$$
A_{r r}=A_{r r}\left(\sum_{s=1}^{n} \bar{A}_{s s}\right)=A_{r r} \bar{A}_{r r}=\left(\sum_{s=1}^{n} A_{s s}\right) \bar{A}_{r r}=\bar{A}_{r r} .
$$

Then $A_{r r}$ are real and $A_{r r} A_{s s}=0, A_{r r}{ }^{2}=A_{r r}$. Therefore, we have a real orthogonal $T$ such that

$$
T A_{r r} T^{\prime}=[0, \cdots, 0,1,0, \cdots, 0]
$$

(where the 1 in the brackets is in the $r$ th place). Without loss of generality we assume that

$$
A_{r r}=[0, \cdots, 0,1,0, \cdots, 0]
$$

(where the 1 in brackets is in the $r$ th place).

(4) Now we put

$$
Z=\left(\begin{array}{cc}
0 & e^{i \theta} \\
e^{i \theta} & 0
\end{array}\right)+[1, \cdots, 1]
$$

From (48) and $I=W \bar{W}$, we have

$$
\begin{aligned}
A_{12} \bar{A}_{12}+e^{i \theta} A_{12}\left(I-A_{11}-A_{22}\right)+\left(I-A_{11}-A_{22}\right) e^{-i \theta} \bar{A}_{12} \\
+\left(I-A_{11}-A_{22}\right)^{2}=I,
\end{aligned}
$$

for all $\theta$. Thus we have

(19) Journal de Mathématique (9) vol. 10 (1931) Theorem 6. 
where $\alpha \bar{\alpha}=I^{(2)}$.

$$
A_{12}=\alpha^{(2)}+0 \text {, }
$$

(5) Further we let

$$
Z=\left(\begin{array}{cc}
e^{i \tau} / 2^{1 / 2} & 1 / 2^{1 / 2} \\
1 / 2^{1 / 2} & -e^{-i \tau} / 2^{1 / 2}
\end{array}\right)+0^{(n-2)} .
$$

From (48) and $I-W \bar{W}=0$, we deduce immediately that

$$
\begin{aligned}
I^{(2)} & =2^{-1}\left(\left(\begin{array}{cc}
e^{i \tau} & 0 \\
0 & -e^{-i \tau}
\end{array}\right)+\alpha\right)\left(\left(\begin{array}{cc}
e^{-i \tau} & 0 \\
0 & -e^{i \tau}
\end{array}\right)+\bar{\alpha}\right) \\
& =2^{-1} I^{(2)}+2^{-1}\left(\alpha\left(\begin{array}{cc}
e^{-i \tau} & 0 \\
0 & -e^{i \tau}
\end{array}\right)+\left(\begin{array}{cc}
e^{i \tau} & 0 \\
0 & -e^{-i \tau}
\end{array}\right) \bar{\alpha}\right)+2^{-1} \alpha \bar{\alpha} .
\end{aligned}
$$

Consequently

$$
\alpha\left(\begin{array}{cc}
e^{-i \tau} & 0 \\
0 & -e^{i \tau}
\end{array}\right)+\left(\begin{array}{cc}
e^{i \tau} & 0 \\
0 & -e^{-i \tau}
\end{array}\right) \bar{\alpha}=0
$$

for all $\tau$. Then

$$
\alpha= \pm\left(\begin{array}{ll}
0 & 1 \\
1 & 0
\end{array}\right)
$$

Similarly $A_{r s}$ is determined completely apart from a sign. We may assume that $A_{1}$ takes the positive sign. Since

$$
\left(\begin{array}{lll}
z_{11} & z_{12} & z_{13} \\
z_{12} & z_{22} & z_{23} \\
z_{13} & z_{23} & z_{33}
\end{array}\right)
$$

being unitary does not imply that

$$
\left(\begin{array}{ccc}
z_{11} & z_{12} & z_{13} \\
z_{12} & z_{22} & -z_{23} \\
z_{13} & -z_{23} & z_{33}
\end{array}\right)
$$

is unitary, the theorem is now established.

Finally, the author gives the following theorem which shows the importance of the notion of the characteristic roots of the distance-matrix.

THEOREM 15. A mapping carrying the hyperbolic space " $I-Z \bar{Z}$ being positive definite" into itself, and keeping the characteristic roots of the distance matrix between two points invariant, is either a hyperbolic motion or a hyperbolic motion combined with a reflexion $Z=\bar{W}$.

Proof. The proof is comparatively simple, and it contains some repetition of our old argument. The author gives only the main procedure of the proof. 
(1) The distance matrix $\mathfrak{D}(A, B)$ of two points $A$ and $B$ of the space is defined by

$$
\mathfrak{D}(A, B)=(A-B)\left(A-\bar{B}^{-1}\right)^{-1}\left(\left(\bar{A}^{-1}-B\right)\left(\bar{A}^{-1}-\bar{B}^{-1}\right)^{-1}\right)^{-1} .
$$

In particular for $B=0$, we have

$$
\mathfrak{D}(A, 0)=A \bar{A}^{\prime} .
$$

(2) Owing to the transitivity, we need only consider the mapping $W=\Gamma(Z)$ keeping 0 invariant. Thus both Hermitian matrices $W \bar{W}$ and $Z \bar{Z}$ are conjunctive. In particular the mapping keeps the rank of $Z$ invariant.

(3) Let

$$
\Gamma([1 / 2,0, \cdots, 0])=\left(a_{1}, \cdots, a_{n}\right)^{\prime}\left(a_{1}, \cdots, a_{n}\right) .
$$

By a slight modification, we may let

$$
\Gamma([1 / 2,0, \cdots, 0])=[1 / 2,0, \cdots, 0] .
$$

From the invariance of the characteristic roots of distance-matrix we find

$$
\Gamma\left(\left[\lambda_{1}, 0, \cdots, 0\right]\right)=\left[\lambda_{1}, 0, \cdots, 0\right],
$$

for all real $\lambda_{1}$. Without loss of generality, we may modify $\Gamma$ such that

$$
\begin{aligned}
& \Gamma\left(\left[0, \lambda_{2}, 0, \cdots, 0\right]\right)=\left[0, \lambda_{2}, 0, \cdots, 0\right], \\
& \left.\Gamma\left(\left[0,0, \cdots, 0, \lambda_{n}\right]\right)=\left[0,0, \cdots, 0, \lambda_{n}\right]\right)
\end{aligned}
$$

for all real $\lambda_{2}, \cdots, \lambda_{\Delta}$.

Next, we may show that for any real diagonal $\Lambda$ we have

$$
\Gamma(\Lambda)=\Lambda,
$$

and that for all real $X$, we have

$$
\Gamma(X)=X .
$$

(4) Since $W=\Gamma(Z)$ and

$$
r(X, W)=r(X, Z),
$$

we find that

$$
|d(X-W)|^{2}=|d(X-Z)|^{2}
$$

for all real $X$. We deduce that

$$
W=Z \text { or } \bar{Z} .
$$

National Tsing Hua University,

Kunming, Yunnan, China. 